\title{
ANTIBACTERIAL Zn(II) COMPOUNDS OF.SCHIFF BASES DERIVED FROM SOME BENZOTHIAZOLES
}

\author{
Mahmood-ul-Hassan', Zahid H. Chohan*' and Claudiu T. Supuran? \\ ${ }^{1}$ Department of Chemistry, Islamia University, Bahawalpur, Pakistan \\ ${ }^{2}$ University of Florence, Dipartimento di Chimica, Laboratorio di Chimica Bioinorganica, Via della \\ Lastruccia 3, Rm 188, Polo Scientifico, 50019-Sesto Fiorentino (Firenze), Italy
}

\begin{abstract}
A condensation reaction of 4-acetamidobenzaldehyde with 2-aminobenzothiazole, 2-amino-4methylbenzothiazole, 2-amino-4-methoxybenzothiazole, 2-amino-4-chlorobenzothiazole, 2-amino-6nitrobenzothiazole and 6-(methylsulfonyl)benzothiazole to form tridentate Schiff bases was used. These Schiff bases have been converted into their $\mathrm{Zn}$ (II) chelates. These Schiff bases and $\mathrm{Zn}$ (II) chelates of the type $\left[\mathrm{M}(\mathrm{L})_{2}\right] \mathrm{Cl}_{2}$ have been characterized by physical, spectral, and analytical data. The Schiff bases act tridentately and are proposed to have octahedral geometry. These compounds have also been screened for their antibacterial properties against pathogenic bacterial species i.e., Escherichia coli, Staphylococcus aureus, and Pseudomonas aeruginosa.
\end{abstract}

\section{INTRODUCTION}

Benzothiazoles are well known biologically active compounds ${ }^{1-4}$. Much research has been focused ${ }^{5-10}$ to highlight the ligational and biological behavior of Schiff bases and their derivatives. The azomethine linkage $(\mathrm{CH}=\mathrm{N})$, is significant feature that makes them interesting candidates ${ }^{11-13}$ for biological activities as well as in the coordination chemistry. The biological activity of certain compounds is related to their ability ${ }^{14.13}$ to form complexes with the metal ions which may induce through coordination a "lock geometry" of the apoprotein metal binding site so that only certain substances are able to become attached to the framework ${ }^{16,17}$ formed by this interaction. Many of the anticancer drugs are versatile ligands, ${ }^{18}$ some of which exhibit increased anticancer activity when administered in the form of their metal complexes ${ }^{10,20}$. It has been suggested ${ }^{22}$ despite some controversy that certain types of cancers are virus-caused ${ }^{21}$. The interaction between the metal ion and the ligand with cancer-associated viruses might represent an important route in designing new anticancer therapies ${ }^{23}$. The inverse process, i.e., coordinating a metal ion from an important biomolecule, for instance a zinc finger protein, has recently been used to design novel antiviral therapies, targeted against virus-causing infections ${ }^{24.25}$. All these observations and the essential role of azomethine linkage attracted our attention to synthesize some benzothiazole derived Schiff bases $\left(\mathrm{HL}^{1}-\mathrm{HL}^{6}\right)$ (Fig 1) and their $\mathrm{Zn}(\mathrm{II})$ chelates (Table 2) and to study their biological behavior via their coordination with the expectation that this alteration may result in achieving new targets in synthesizing and designing of compounds that could fight agressively against antibiotic resistant strains. These synthesized compounds have been characterized by physical, spectral and analytical data and also screened for their antibacterial activities against pathogenic bacterial species i.e., Escherichia coli, Staphylococcus aureus, and Pseudomonas aeruginosa.

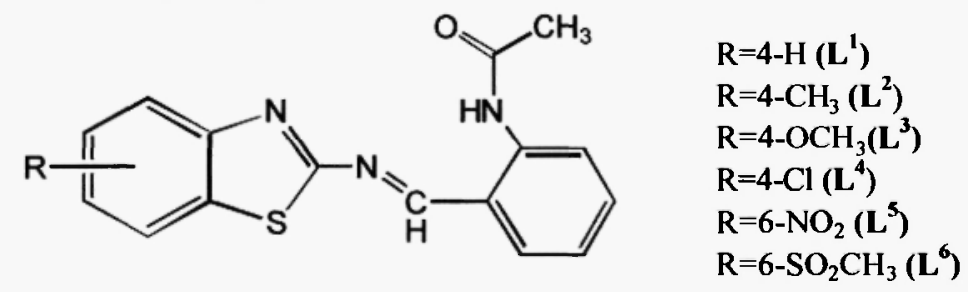

Fig 1. Structure of Schiff bases

\section{EXPERIMENTAL}

\section{Material and Methods}

All chemicals and solvents used were of Analar grade. All metal(II) salts were used as chlorides. IR spectra were recorded on a Philips Analytical PU 9800 FTIR spectrophotometer. UV-Visible spectra were obtained in DMF on a Hitachi U-2000 double-beam spectrophotometer. $\mathrm{C}, \mathrm{H}$ and $\mathrm{N}$ analyses was carried out by Butterworth Laboratories Ltd. Conductance of the metal complexes was determined in DMF on a Hitachi YSI-32 model conductometer. Magnetic measurements were made on solid complexes using the Gouy method. Melting points were recorded on a Gallenkamp apparatus and are uncorrected. 
Preparation of the Schiff base $\left(L^{1}\right)$

2-Aminobenzothiazole $(1.5 \mathrm{~g}, 0.01 \mathrm{M})$ in ethanol $(10 \mathrm{~mL})$ was added to a hot ethanol solution $(30 \mathrm{~mL})$ of 4acetamidobenzaldehyde $(1.6 \mathrm{~g}, 0.01 \mathrm{M})$. Then $2-3$ drops of conc. $\mathrm{H}_{2} \mathrm{SO}_{4}$ were added and the mixture refluxed for $2 \mathrm{~h}$. On cooling, a solid product was formed which was filtered, washed with ethanol, then with ether and dried. Crystallization from hot ethanol gave $L^{1}$. The same method was applied for the preparation of $L^{2}-L^{5}$ by using the corresponding reagents in the same molar ratio.

Preparation of the $Z n(I I)$ complex of $L^{1}$

A warm ethanol solution $(20 \mathrm{~mL})$ of $\mathrm{L}^{1}(0.02 \mathrm{M})$ was added to a magnetically stirred solution of cobalt chloride hexahydrate $(0.01 \mathrm{M})$ in distilled water $(25 \mathrm{~mL})$. The mixture was refluxed for $1 \mathrm{~h}$ and cooled to room temperature. On cooling, a precipitates was formed which were filtered, washed with ethanol, acetone and ether, and dried by suction. Crystallization from aqueous ethanol (30:70) gave the desired metal complex (1). All other $\mathrm{Zn}(\mathrm{II})$ complexes of $\mathrm{L}^{2}-\mathrm{L}^{6}$ were prepared respectively following the same method.

Antibacterial studies

The synthesized $\mathrm{Zn}(\mathrm{II})$ complexes and the uncomplexed Schiff bases were screened for their antibacterial activity against pathogenic bacterial strains, Escherichia coli, Staphylococcus aureus and Pseudomonas aeruginosa. The paper disc diffusion method ${ }^{26}$ was adopted for the determination of antibacterial activity.

\section{RESULTS AND DISCUSSION}

Physical properties

The Schiff bases $\left(\mathrm{L}^{1}-\mathrm{L}^{6}\right)$ (Fig. 1) were prepared by refluxing an appropriate amount of 4 acetamidobenzaldehyde with 2-aminobenzothiazole and its substituted 4-methyl, 4-methoxy-, 4-chloro-, 6nitro- and 6-(methylsulfonyl)benzothiazole in hot ethanol in 1:1 molar ratio respectively. The structures of these Schiff bases formed were established with the help of their IR, NMR, and microanalytical data (Tables 1 and 3)

Table I. Physical, Spectral and Analytical Data of the Schiff bases.

\begin{tabular}{|c|c|c|c|c|}
\hline Schiff base & IR $\left(\mathrm{cm}^{-1}\right)$ & $\begin{array}{l}\text { Calc (Found) \% } \\
\mathrm{C} \quad \mathrm{H} N\end{array}$ & $\begin{array}{l}\text { M.P } \\
\left({ }^{\circ} \mathrm{C}\right)\end{array}$ & $\begin{array}{c}\text { Yield } \\
(\%)\end{array}$ \\
\hline $\begin{array}{c}\mathrm{L}^{\mathrm{I}} \\
\mathrm{C}_{16} \mathrm{H}_{13} \mathrm{~N}_{3} \mathrm{OS} \\
{[295.0]}\end{array}$ & $\begin{array}{l}3190(\mathrm{~ms}, \mathrm{NH}), 1685,1540 \\
(\mathrm{~s}, \quad \mathrm{CONH}), \quad 1635 \\
\mathrm{HC}=\mathrm{N}), 1615(\mathrm{~s}, \mathrm{C}=\mathrm{N}) .\end{array}$ & $\begin{array}{ccc}65.1 & 4.4 & 14.2 \\
(65.5)(4.5)(14.0)\end{array}$ & 123 & 68 \\
\hline $\begin{array}{c}\mathrm{L}^{2} \\
\mathrm{C}_{17} \mathrm{H}_{15} \mathrm{~N}_{3} \mathrm{OS} \\
{[309.0]}\end{array}$ & $\begin{array}{l}3190(\mathrm{~ms}, \mathrm{NH}), 1685,1540 \\
(\mathrm{~s}, \mathrm{CONH}), \quad 1630 \quad(\mathrm{~s}, \\
\mathrm{HC}=\mathrm{N}), 1615(\mathrm{~s}, \mathrm{C}=\mathrm{N}) .\end{array}$ & $\begin{array}{ccc}66.0 & 4.9 & 13.6 \\
(66.3)(4.5)(13.8)\end{array}$ & 137 & 62 \\
\hline $\begin{array}{c}\mathrm{L}^{\mathrm{J}} \\
\mathrm{C}_{17} \mathrm{H}_{15} \mathrm{~N}_{3} \mathrm{O}_{2} \mathrm{~S} \\
{[325.0]}\end{array}$ & $\begin{array}{l}3190(\mathrm{~ms}, \mathrm{NH}), 1685,1540 \\
(\mathrm{~s}, \quad \mathrm{CONH}), \quad 1635 \\
\mathrm{HC}=\mathrm{N}), 1615(\mathrm{~s}, \mathrm{C}=\mathrm{N}) .\end{array}$ & $\begin{array}{ccc}62.8 & 4.6 & 12.9 \\
(62.5)(4.9)(12.5)\end{array}$ & 126 & 70 \\
\hline $\begin{array}{c}\mathrm{L}_{16}^{4} \mathrm{H}_{12} \mathrm{ClN}_{3} \mathrm{OS} \\
{[329.5]}\end{array}$ & $\begin{array}{l}3190(\mathrm{~ms}, \mathrm{NH}), 1685,1540 \\
(\mathrm{~s}, \mathrm{CONH}), \quad 1635 \quad(\mathrm{~s}, \\
\mathrm{HC}=\mathrm{N}), 1615(\mathrm{~s}, \mathrm{C}=\mathrm{N}), 720 \\
(\mathrm{~m}, \mathrm{C}-\mathrm{Cl}) .\end{array}$ & $\begin{array}{ccc}58.3 & 3.6 & 12.7 \\
(58.5)(3.9)(12.4)\end{array}$ & 162 & 65 \\
\hline $\begin{array}{c}\mathrm{L}^{3} \\
\mathrm{C}_{16} \mathrm{H}_{12} \mathrm{~N}_{4} \mathrm{O}_{3} \mathrm{~S} \\
{[340.0]}\end{array}$ & $\begin{array}{l}3190(\mathrm{~ms}, \mathrm{NH}), 1685,1540 \\
(\mathrm{~s}, \quad \mathrm{CONH}), \quad 1635 \\
\mathrm{HC}=\mathrm{N}), 1615(\mathrm{~s}, \mathrm{C}=\mathrm{N}) .\end{array}$ & $\begin{array}{ccc}56.5 & 3.5 & 16.5 \\
(56.9)(3.2)(16.3)\end{array}$ & 148 & 67 \\
\hline $\begin{array}{c}\mathrm{L}^{6} \\
\mathrm{C}_{17} \mathrm{H}_{15} \mathrm{~N}_{3} \mathrm{O}_{3} \mathrm{~S}_{2} \\
{[373.0]}\end{array}$ & $\begin{array}{l}3190(\mathrm{~ms}, \mathrm{NH}), 1685,1540 \\
(\mathrm{~s}, \mathrm{CONH}), \quad 1635 \quad(\mathrm{~s}, \\
\mathrm{HC}=\mathrm{N}), \quad 1615 \quad(\mathrm{~s}, \mathrm{C}=\mathrm{N}), \\
1380\left(\mathrm{~s}, \mathrm{SO}_{2}\right) .\end{array}$ & $\begin{array}{ccc}54.7 & 4.0 & 11.3 \\
(54.8)(4.2)(11.1)\end{array}$ & 155 & 65 \\
\hline
\end{tabular}

$\mathrm{s}=$ sharp, $\mathrm{ms}=$ medium sharp

These Schiff bases were then used for the complexation with $\mathrm{Zn}(\mathrm{II})$ ion. All of the synthesized metal complexes [(1)-(6)] (Table 2) were air and moisture stable. These were prepared by the stoichiometric reaction of the corresponding $\mathrm{Zn}$ (II) metal salt (as chloride) and the Schiff base in molar ratios M:L of 1:2. The complexes are amorphous solids, which decompose above $200^{\circ} \mathrm{C}$. They are insoluble in common organic solvents such as ethanol, methanol, chloroform or acetone, but soluble in DMSO and DMF. Molar conductance values of the soluble complexes in DMF showed low values $\left(72-76 \mathrm{ohm}^{-1} \mathrm{~cm}^{2} \mathrm{~mol}^{-1}\right)$ indicating them to be be electrolytes.

\section{Infrared spectra}

IR spectra of the Schiff bases showed the absence of bands at 1735 and $3420 \mathrm{~cm}^{-1}$ due to carbonyl $v(\mathrm{C}=\mathrm{O})$ and $v\left(\mathrm{NH}_{2}\right)$ stretching vibrations and, instead, appearance of a strong new band at $\sim 1635 \mathrm{~cm}^{-1}$ assigned ${ }^{28}$ to the azomethine $v(\mathrm{HC}=\mathrm{N})$ linkage. This suggested that amino and aldehyde moieties of the starting reagents no more existed and have been converted into their corresponding Schiff bases (Fig.l). The comparison of 
the IR spectra of the Schiff bases and their $\mathrm{Zn}$ (II) chelates indicated that the Schiff bases were coordinated to the metal atom in three ways, thus representing the ligands acting in a tridentate manner.

Table 2. Physical, Spectral and Analytical Data of the $\mathrm{Zn}$ (II) Chelates.

\begin{tabular}{|c|c|c|c|c|c|}
\hline $\begin{array}{l}\text { Metal chelate/ } \\
\text { Mol. Formula }\end{array}$ & $\begin{array}{l}\text { Yield } \\
(\%)\end{array}$ & $\begin{array}{l}\text { M.P } \\
\left({ }^{\circ} \mathrm{C}\right)\end{array}$ & $\operatorname{IR~}\left(\mathrm{cm}^{-1}\right)$ & $\begin{array}{l}\lambda_{\max } \\
\left(\mathrm{cm}^{-1}\right)\end{array}$ & $\begin{array}{l}\text { Calc (Found)\% } \\
\mathrm{C} \quad \mathrm{H} N\end{array}$ \\
\hline 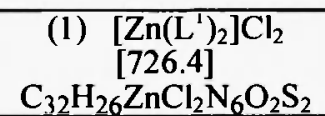 & 60 & $\begin{array}{l}208- \\
210\end{array}$ & $\begin{array}{l}1625 \quad(\mathrm{~s}, \mathrm{HC}=\mathrm{N}), 1600 \quad(\mathrm{~s}, \\
\mathrm{C}=\mathrm{N}), 1670,1535(\mathrm{~s}, \mathrm{CONH}) \\
530(\mathrm{~ms}, \mathrm{M}-\mathrm{N}), 455(\mathrm{~ms}, \mathrm{M}-\mathrm{O})\end{array}$ & 27,445 & $\begin{array}{lll}52.9 & 3.6 & 11.6 \\
(53.2)(3.2)(118)\end{array}$ \\
\hline $\begin{array}{c}(2)\left[\mathrm{Zn}(\mathrm{L})_{2}\right] \mathrm{Cl}_{2} \\
{[754.4]^{2}} \\
\mathrm{C}_{34} \mathrm{H}_{30} \mathrm{ZnCl}_{2} \mathrm{~N}_{6} \mathrm{O}_{2} \mathrm{~S}_{2}\end{array}$ & 60 & $\begin{array}{l}215- \\
217\end{array}$ & $\begin{array}{l}1625 \quad(\mathrm{~s}, \quad \mathrm{HC}=\mathrm{N}), \quad 1595 \quad(\mathrm{~s}, \\
\mathrm{C}=\mathrm{N}), 1675,1535(\mathrm{~s}, \mathrm{CONH}) \\
530(\mathrm{~ms}, \mathrm{M}-\mathrm{N}), 460(\mathrm{~ms}, \mathrm{M}-\mathrm{O})\end{array}$ & 27,415 & $\begin{array}{lll}54.1 & 4.0 & 11.1 \\
(54.5)(4.4)(11.0)\end{array}$ \\
\hline $\begin{array}{c}\text { (3) }\left[\mathrm{Zn}\left(\mathrm{L}^{3}\right)_{2}\right] \mathrm{Cl}_{2} \\
{[786.4]} \\
\mathrm{C}_{34} \mathrm{H}_{30} \mathrm{ZnCl}_{2} \mathrm{~N}_{6} \mathrm{O}_{4} \mathrm{~S}_{2}\end{array}$ & 58 & $\begin{array}{l}202- \\
204\end{array}$ & $\begin{array}{l}1620 \quad(\mathrm{~s}, \quad \mathrm{HC}=\mathrm{N}), \quad 1600(\mathrm{~s}, \\
\mathrm{C}=\mathrm{N}), 1675,1530(\mathrm{~s}, \mathrm{CONH}), \\
525(\mathrm{~ms}, \mathrm{M}-\mathrm{N}), 460(\mathrm{~ms}, \mathrm{M}-\mathrm{O})\end{array}$ & 27,410 & $\begin{array}{lll}51.9 & 3.8 & 10.7 \\
(51.7)(3.5)(10.9)\end{array}$ \\
\hline $\begin{array}{c}\text { (4) }\left[\mathrm{Zn}\left(\mathrm{L}^{4}\right)_{2}\right] \mathrm{Cl}_{2} \\
{[7599.9]} \\
\mathrm{C}_{32} \mathrm{H}_{24} \mathrm{ZnCl}_{3} \mathrm{~N}_{6} \mathrm{O}_{2} \mathrm{~S}_{2}\end{array}$ & 61 & $\begin{array}{l}218- \\
220\end{array}$ & $\begin{array}{l}1625 \quad(\mathrm{~s}, \mathrm{HC}=\mathrm{N}), 1595 \quad(\mathrm{~s}, \\
\mathrm{C}=\mathrm{N}), 1675,1535(\mathrm{~s}, \mathrm{CONH}), \\
525(\mathrm{~ms}, \mathrm{M}-\mathrm{N}), 455(\mathrm{~ms}, \mathrm{M}-\mathrm{O})\end{array}$ & 27,425 & $\begin{array}{lll}50.5 & 3.2 & 11.1 \\
(50.7)(3.3)(11.5)\end{array}$ \\
\hline $\begin{array}{c}\text { (5) }\left[\mathrm{Zn}\left(\mathrm{L}^{3}\right)_{2}\right] \mathrm{Cl}_{2} \\
{[816.4]} \\
\mathrm{C}_{32} \mathrm{H}_{24} \mathrm{ZnCl}_{2} \mathrm{~N}_{8} \mathrm{O}_{6} \mathrm{~S}_{2}\end{array}$ & 61 & $\begin{array}{l}211- \\
213\end{array}$ & $\begin{array}{l}1625 \quad(\mathrm{~s}, \mathrm{HC}=\mathrm{N}), 1595(\mathrm{~s}, \\
\mathrm{C}=\mathrm{N}), 1675,1535(\mathrm{~s}, \mathrm{CONH}) \\
525(\mathrm{~ms}, \mathrm{M}-\mathrm{N}), 455(\mathrm{~ms}, \mathrm{M}-\mathrm{O})\end{array}$ & 27,425 & $\begin{array}{lll}47.0 & 2.9 & 13.7 \\
(47.4)(2.5)(13.5)\end{array}$ \\
\hline $\begin{array}{c}\text { (6) }\left[\mathrm{Zn}\left(\mathrm{L}^{\circ}\right)_{2}\right] \mathrm{Cl}_{2} \\
{[882.4]} \\
\mathrm{C}_{34} \mathrm{H}_{30} \mathrm{ZnCl}_{2} \mathrm{~N}_{6} \mathrm{O}_{6} \mathrm{~S}_{4}\end{array}$ & 62 & $\begin{array}{l}220- \\
222\end{array}$ & $\begin{array}{l}1625(\mathrm{~s}, \mathrm{HC}=\mathrm{N}), 1595(\mathrm{~s}, \\
\mathrm{C}=\mathrm{N}), 1675,1535(\mathrm{~s}, \mathrm{CONH}), \\
525(\mathrm{~ms}, \mathrm{M}-\mathrm{N}), 455(\mathrm{~ms}, \mathrm{M}-\mathrm{O})\end{array}$ & 27,465 & $\begin{array}{l}46.2 \quad 3.4 \quad 9.5 \\
(46.6)(3.3)(9.7)\end{array}$ \\
\hline
\end{tabular}

$\mathrm{s}=$ sharp, $\mathbf{m s}=$ medium sharp

The band appearing at $1635 \mathrm{~cm}^{-1}$ due to the azomethine was shifted to lower frequency by $\sim 10-15 \mathrm{~cm}^{-1}$ indicating ${ }^{29}$ participation of the azomethine nitrogen in the complexation.

The band at $1615 \mathrm{~cm}^{-1}$ assigned to the benzothiazole ring $v(C=N)$ nitrogen also shifted to lower frequency by $-15-20 \mathrm{~cm}^{-1}$ which was indicative of the involvement of ring nitrogen of the benzothiazole moiety in chelation.

A medium strong band appearing at $3190 \mathrm{~cm}^{-1}$ and assigned to $v(\mathrm{NH})$ remained unchanged providing thus a clue that the $\mathrm{NH}$ group in not involved in the coordination. However, bands at 1685 and $1540 \mathrm{~cm}^{-1}$ assigned ${ }^{28}$ to amido group $v(-\mathrm{CONH})$ in the Schiff bases were not found at the same frequencies in the spectra of their $\mathrm{Zn}$ (II) complexes but shifted to lower frequency by $10-15 \mathrm{~cm}^{-1}$ indicating, in turn, the coordination of the amido oxygen $v(-\mathrm{C}=\mathrm{O})$ to the metal atom.

Further conclusive evidence of the coordination of these Schiff bases with the $\mathrm{Zn}(\mathrm{II})$ metal atom was shown by the appearance of weak low-frequency new bands at $525-530$ and $455-460 \mathrm{~cm}$. These were assigned ${ }^{30}$ to the metal-nitrogen $v(\mathrm{M}-\mathrm{N})$ and metal-oxygen $v(\mathrm{M}-\mathrm{O})$ respectively. These new bands were observable only in the spectra of the metal complexes and not in the spectra of its uncomplexed Schiff bases, thus confirming the participation of these hetero groups $(\mathrm{O}$ or $\mathrm{N})$ in the complexation.

\section{NMR spectra}

The ${ }^{1} \mathrm{H}$ NMR spectra of the Schiff bases and of their $\mathrm{Zn}(\mathrm{II})$ complexes taken in DMSO- $\mathrm{d}_{6}$ are listed in Table 3 . The Schiff bases exhibited signals due to all the expected protons in their expected region and have been identified from the integration curve found to be equivalent to the total number of protons deduced from the proposed structures. These were compared with the reported ${ }^{31}$ signals of the known comparable compounds and give further support for the compositions of the new ligands as well as their complexes suggested by their IR and elemental analyses data. Comparison of the chemical shifts of the uncomplexed Schiff bases with those of the corresponding $\mathrm{Zn}$ (II) complexes show that some of the resonance signals underwent a shift upon the complexation. In each case, the protons assigned due to aromatic and azomethine $(\mathrm{HC}=\mathrm{N})$ moieties were found at $\sim 7.1-8.1$ and $6.8 \mathrm{ppm}$ in the spectra of the Schiff bases. The protons due to azomethine and aromatic groups undergo a downfield shift of $0.9-1.0 \mathrm{ppm}$ in the complexes indicating coordination of these groups with the Zn(II) metal atom. ${ }^{13} \mathrm{C}$ NMR spectra likewise showed similar diagnostic features ${ }^{32}$ for the Schiff bases as well as their $\mathrm{Zn}(\mathrm{II})$ complexes.

\section{UV-Visible Spectra}

UV-Visible spectral bands of the $\mathrm{Zn}$ (II) complexes are recorded in Table 4 . The diamagnetic $\mathrm{Zn}$ (II) complexes did not show any $d-d$ bands and their spectra are dominated only by charge transfer bands. The charge transfer band at $27,410-27,465 \mathrm{~cm}^{-1}$ was assigned ${ }^{33}$ to the transition ${ }^{2} \mathrm{E}_{\mathrm{g}} \stackrel{2}{ }^{2} \mathrm{~T}_{2 \mathrm{~g}}$, possibly in an octahedral environment ${ }^{34}$. 
Table 3. ${ }^{1} \mathrm{H}$ and ${ }^{13} \mathrm{C}$ NMR Data of the Schiff bases and its $\mathrm{Zn}$ (II) Complexes.

\begin{tabular}{|c|c|c|}
\hline $\begin{array}{l}\text { Schiff base/ } \\
\text { Complex }\end{array}$ & $\begin{array}{c}{ }^{\mathrm{I}} \mathrm{HNMR} \\
\left(\mathrm{DMSO}-\mathrm{d}_{6}\right) \mathrm{ppm}\end{array}$ & $\begin{array}{c}{ }^{\mathrm{ij}} \mathrm{C} \text { NMR } \\
\left(\mathrm{DMSO}-\mathrm{d}_{6}\right) \mathrm{ppm}\end{array}$ \\
\hline $\mathrm{L}^{1}$ & $\begin{array}{l}2.4\left(\mathrm{~s}, 3 \mathrm{H}, \mathrm{CH}_{3}\right), 6.8(\mathrm{~s}, 1 \mathrm{H}, \mathrm{CH}=\mathrm{N}), \\
7.1-7.2(\mathrm{~m}, 2 \mathrm{H}, \text { aromatic }), 7.3-7.4(\mathrm{~m}, 2 \mathrm{H} \text {, } \\
\text { aromatic), 7.6-7.7 (m, 2H, aromatic), 7.9- } \\
8.1(\mathrm{~m}, 2 \mathrm{H} \text {, aromatic), } 8.3(\mathrm{~s}, 1 \mathrm{H}, \mathrm{NH}) .\end{array}$ & $\begin{array}{l}45.6\left(\mathrm{CH}_{3}\right), 110.8,115.5,120.3,122.8,124.6, \\
125.5, \quad 127.2,128.3,130.3,139.2,142.7, \\
148.6(\text { aromatic }), 126.7(\mathrm{C}-\mathrm{N}), 150.7(\mathrm{C}=\mathrm{N}), \\
192.3(\mathrm{C}=\mathrm{O}) .\end{array}$ \\
\hline $\mathbf{L}^{2}$ & $\begin{array}{l}1.9\left(\mathrm{~s}, 3 \mathrm{H}, \mathrm{CH}_{3}\right), 2.4\left(\mathrm{~s}, 3 \mathrm{H}, \mathrm{CH}_{3}\right), 6.8(\mathrm{~s}, \\
1 \mathrm{H}, \mathrm{CH}=\mathrm{N}), 7.1-7.2(\mathrm{~m}, 1 \mathrm{H}, \text { aromatic), } \\
7.3-7.4(\mathrm{~m}, 2 \mathrm{H}, \text { aromatic }), 7.6-7.7(\mathrm{~m}, 2 \mathrm{H}, \\
\text { aromatic), } 7.9-8.0(\mathrm{~m}, 2 \mathrm{H}, \text { aromatic), } \\
8.3(\mathrm{~s}, 1 \mathrm{H}, \mathrm{NH}) .\end{array}$ & $\begin{array}{l}23.2\left(\mathrm{CH}_{3}\right), 45.6\left(\mathrm{CH}_{3}\right), 111.7,116.2,120.5, \\
122.8,124.6,125.7,127.2,128.3,130.3, \\
139.2,142.7,148.6(\text { aromatic }), 126.7(\mathrm{C}-\mathrm{N}), \\
150.7(\mathrm{C}=\mathrm{N}), 192.3(\mathrm{C}=\mathrm{O}) .\end{array}$ \\
\hline $\mathrm{L}^{3}$ & $\begin{array}{l}3.1\left(\mathrm{~s}, 3 \mathrm{H}, \mathrm{OCH}_{3}\right), 2.4\left(\mathrm{~s}, 3 \mathrm{H}, \mathrm{CH}_{3}\right), 6.8(\mathrm{~s}, \\
1 \mathrm{H}, \mathrm{CH}=\mathrm{N}), 7.2-7.3 \mathrm{~m}, 1 \mathrm{H}, \text { aromatic) } \\
7.4-7.6(\mathrm{~m}, 2 \mathrm{H}, \text { aromatic }), 7.7-7.8(\mathrm{~m}, 2 \mathrm{H}, \\
\text { aromatic), 7.9-8.0 (m, 2H, aromatic), } 8.3 \\
(\mathrm{~s}, 1 \mathrm{H}, \mathrm{NH}) .\end{array}$ & $\begin{array}{lllll}55.3\left(\mathrm{OCH}_{3}\right), & 45.6\left(\mathrm{CH}_{3}\right), & 111.8, & 116.3, \\
120.6, \quad 122.8, \quad 124.6, \quad 125.5, & 127.2, & 128.3, \\
130.3, & 139.2,142.7,148.6(\text { aromatic }), & 126.7 \\
(\mathrm{C}-\mathrm{N}), 150.7(\mathrm{C}=\mathrm{N}), 192.3(\mathrm{C}=\mathrm{O}) .\end{array}$ \\
\hline $\mathrm{L}^{4}$ & $\begin{array}{l}2.4\left(\mathrm{~s}, 3 \mathrm{H}, \mathrm{CH}_{3}\right), 6.8(\mathrm{~s}, 1 \mathrm{H}, \mathrm{CH}=\mathrm{N}), \\
7.2-7.3(\mathrm{~m}, 1 \mathrm{H}, \text { aromatic }), 7.4-7.6(\mathrm{~m}, 2 \mathrm{H}, \\
\text { aromatic), 7.7-7.8 (m, 2H, aromatic), 7.9- } \\
8.1(\mathrm{~m}, 2 \mathrm{H}, \text { aromatic }), 8.3(\mathrm{~s}, 1 \mathrm{H}, \mathrm{NH}) .\end{array}$ & $\begin{array}{l}45.6\left(\mathrm{CH}_{3}\right), 111.9,116.7,120.4,122.8,124.6, \\
125.6, \quad 127.2,128.3,130.3,139.2,142.7, \\
148.6(\text { aromatic }), 126.7(\mathrm{C}-\mathrm{N}), 150.7(\mathrm{C}=\mathrm{N}), \\
192.3(\mathrm{C}=\mathrm{O}) \text {. }\end{array}$ \\
\hline $\mathrm{L}^{5}$ & $\begin{array}{l}2.4\left(\mathrm{~s}, 3 \mathrm{H}, \mathrm{CH}_{3}\right), 6.8(\mathrm{~s}, 1 \mathrm{H}, \mathrm{CH}=\mathrm{N}), \\
7.2-7.3(\mathrm{~m}, 1 \mathrm{H}, \text { aromatic }), 7.4-7.6(\mathrm{~m}, 2 \mathrm{H} \text {, } \\
\text { aromatic }), 7.7-7.8(\mathrm{~m}, 2 \mathrm{H}, \text { aromatic }), 7.9- \\
8.1(\mathrm{~m}, 2 \mathrm{H}, \text { aromatic }), 8.3(\mathrm{~s}, 1 \mathrm{H}, \mathrm{NH}) .\end{array}$ & $\begin{array}{l}45.6\left(\mathrm{CH}_{3}\right), 111.8,116.8,120.4,122.8,124.7, \\
125.5,127.3,128.3,130.3,139.3,142.7 \\
148.6(\text { aromatic }), 126.7(\mathrm{C}-\mathrm{N}) .150 .7(\mathrm{C}=\mathrm{N}), \\
192.3(\mathrm{C}=\mathrm{O}) .\end{array}$ \\
\hline $\mathrm{L}^{0}$ & $\begin{array}{l}3.6\left(\mathrm{~s}, 3 \mathrm{H}, \mathrm{SO}_{2} \mathrm{CH}_{3}\right) 2.4\left(\mathrm{~s}, 3 \mathrm{H}, \mathrm{CH}_{3}\right), 6.8 \\
(\mathrm{~s}, 1 \mathrm{H}, \mathrm{CH}=\mathrm{N}), 7.2-7.3(\mathrm{~m}, 1 \mathrm{H}, \text { aromatic), } \\
7.4-7.6(\mathrm{~m}, 2 \mathrm{H}, \text { aromatic), } 7.7-7.8(\mathrm{~m}, 2 \mathrm{H}, \\
\text { aromatic), 7.9-8.1 (m, 2H, aromatic), } 8.3 \\
(\mathrm{~s}, 1 \mathrm{H}, \mathrm{NH}) .\end{array}$ & $\begin{array}{l}58.8\left(\mathrm{SO}_{2} \mathrm{CH}_{3}\right), 45.6 \quad\left(\mathrm{CH}_{3}\right), \quad 111.9,115.5, \quad 124.6, \quad 125.6, \quad 127.2,128.4, \\
120.4,122.9, \quad 124,148.6 \text { (aromatic), } 126.8 \\
130.3,139.3,142.8,148.3(\mathrm{C}=\mathrm{O}) .\end{array}$ \\
\hline (1) & $\begin{array}{l}2.6\left(\mathrm{~s}, 3 \mathrm{H}, \mathrm{CH}_{3}\right), 7.0(\mathrm{~s}, 1 \mathrm{H}, \mathrm{CH}=\mathrm{N}), \\
7.2-7.3(\mathrm{~m}, 2 \mathrm{H}, \text { aromatic }), 7.3-7.4(\mathrm{~m}, 2 \mathrm{H} \text {, } \\
\text { aromatic), 7.6-7.7 (m, 2H, aromatic), 7.9- } \\
8.1(\mathrm{~m}, 2 \mathrm{H} \text {, aromatic), } 8.4(\mathrm{~s}, 1 \mathrm{H}, \mathrm{NH}) .\end{array}$ & $\begin{array}{l}45.6\left(\mathrm{CH}_{3}\right), 110.9,115.5,120.4,122.8,124.7, \\
125.5,127.2,128.4,130.3,139.3,142.7, \\
148.7 \text { (aromatic), 126.7 (C-N), 150.9 (C=N), } \\
192.5(\mathrm{C}=\mathrm{O}) \text {. }\end{array}$ \\
\hline (2) & $\begin{array}{l}1.9\left(\mathrm{~s}, 3 \mathrm{H}, \mathrm{CH}_{3}\right), 2.6\left(\mathrm{~s}, 3 \mathrm{H}, \mathrm{CH}_{3}\right), \\
1 \mathrm{H}, \mathrm{CH}=\mathrm{N}), 7.2-7.3(\mathrm{~m}, 1 \mathrm{H}, \text { aror } \\
7.4-7.5(\mathrm{~m}, 2 \mathrm{H}, \text { aromatic), 7.6-7.7 (n } \\
\text { aromatic), 7.9-8.0 (m, 2H, aromatic } \\
(\mathrm{s}, 1 \mathrm{H}, \mathrm{NH}) .\end{array}$ & $\begin{array}{l}23.2\left(\mathrm{CH}_{3}\right), 45.6\left(\mathrm{CH}_{3}\right), 111.8, \\
116.3,120.5,122.8,124.6,125.8,127.2 \text {, } \\
128.4,130.3,139.2,142.7,148.6(\text { aromatic }), \\
126.7(\mathrm{C}-\mathrm{N}), 150.9(\mathrm{C}=\mathrm{N}), 192.5(\mathrm{C}=\mathrm{O}) .\end{array}$ \\
\hline (3) & $\begin{array}{l}3.1\left(\mathrm{~s}, 3 \mathrm{H}, \mathrm{OCH}_{3}\right), 2.6\left(\mathrm{~s}, 3 \mathrm{H}, \mathrm{CH}_{3}\right), 7.0(\mathrm{~s}, \\
1 \mathrm{H}, \mathrm{CH}=\mathrm{N}), 7.3-7.4(\mathrm{~m}, 1 \mathrm{H}, \text { aromatic), } \\
7.5-7.6(\mathrm{~m}, 2 \mathrm{H}, \text { aromatic), 7.7-7.8 (m, } 2 \mathrm{H}, \\
\text { aromatic), 7.9-8.0 (m, 2H, aromatic), } 8.4 \\
(\mathrm{~s}, 1 \mathrm{H}, \mathrm{NH}) .\end{array}$ & $\begin{array}{lllll}55.3\left(\mathrm{OCH}_{3}\right), 45.6\left(\mathrm{CH}_{3}\right), & 111.8, & 116.4, \\
120.6, \quad 122.8, & 124.7, \quad 125.5, & 127.2, & 128.4 \\
130.3,139.3,142.7, & 148.6(\text { aromatic }), & 126.7 \\
(\mathrm{C}-\mathrm{N}), 150.8(\mathrm{C}=\mathrm{N}), 192.5(\mathrm{C}=\mathrm{O}) . & \end{array}$ \\
\hline (4) & $\begin{array}{l}2.6\left(\mathrm{~s}, 3 \mathrm{H}, \mathrm{CH}_{3}\right), 7.0(\mathrm{~s}, 1 \mathrm{H}, \mathrm{CH}=\mathrm{N}), \\
7.2-7.3(\mathrm{~m}, 1 \mathrm{H}, \text { aromatic), } 7.5-7.7(\mathrm{~m}, 2 \mathrm{H} \text {, } \\
\text { aromatic), 7.8-7.9 (m, 2H, aromatic), 8.0- } \\
8.1(\mathrm{~m}, 2 \mathrm{H}, \text { aromatic), } 8.4(\mathrm{~s}, 1 \mathrm{H}, \mathrm{NH}) .\end{array}$ & $\begin{array}{l}45.6\left(\mathrm{CH}_{3}\right), 111.9,116.8,120.4,122.8,124.7, \\
125.6,127.2,128.4,130.4,139.2,142.8, \\
148.6(\text { aromatic }), 126.7(\mathrm{C}-\mathrm{N}), 150.9(\mathrm{C}=\mathrm{N}) \text {, } \\
192.5(\mathrm{C}=\mathrm{O}) .\end{array}$ \\
\hline$(5)$ & $\begin{array}{l}2.6\left(\mathrm{~s}, 3 \mathrm{H}, \mathrm{CH}_{3}\right), 7.0(\mathrm{~s}, 1 \mathrm{H}, \mathrm{CH}=\mathrm{N}), \\
7.3-7.4(\mathrm{~m}, 1 \mathrm{H}, \text { aromatic), } 7.5-7.6(\mathrm{~m}, 2 \mathrm{H} \text {, } \\
\text { aromatic }), 7.7-7.8(\mathrm{~m}, 2 \mathrm{H}, \text { aromatic), 7.9- } \\
8.1(\mathrm{~m}, 2 \mathrm{H}, \text { aromatic }), 8.4(\mathrm{~s}, 1 \mathrm{H}, \mathrm{NH}) .\end{array}$ & $\begin{array}{l}45.6\left(\mathrm{CH}_{3}\right), 111.8,116.9,120.4,122.9,124.7, \\
125.5,127.4,128.3,130.4,139.3,142.8, \\
148.6(\text { aromatic }), 126.7(\mathrm{C}-\mathrm{N}), 150.9(\mathrm{C}=\mathrm{N}), \\
192.5(\mathrm{C}=\mathrm{O}) .\end{array}$ \\
\hline (6) & $\begin{array}{l}3.7\left(\mathrm{~s}, 3 \mathrm{H}, \mathrm{SO}_{2} \mathrm{CH}_{3}\right) 2.6\left(\mathrm{~s}, 3 \mathrm{H}, \mathrm{CH}_{3}\right), 7.0 \\
(\mathrm{~s}, 1 \mathrm{H}, \mathrm{CH}=\mathrm{N}), 7.2-7.3(\mathrm{~m}, 1 \mathrm{H}, \text { aromatic), } \\
7.5-7.7(\mathrm{~m}, 2 \mathrm{H}, \text { aromatic), } 7.8-7.9(\mathrm{~m}, 2 \mathrm{H}, \\
\text { aromatic), 8.0-8.2 (m, 2H, aromatic), } 8.4 \\
(\mathrm{~s}, 1 \mathrm{H}, \mathrm{NH}) .\end{array}$ & $\begin{array}{l}58.9\left(\mathrm{SO}_{2} \mathrm{CH}_{3}\right), 45.6\left(\mathrm{CH}_{7}\right), \quad 111.9,115.6,124.6, \quad 125.6,127.3,128.4, \\
120.5,122.9,124.8,148.6 \text { (aromatic), } 126.8 \\
130.4,139.3,142.8,192.6(\mathrm{C}=\mathrm{O}) .\end{array}$ \\
\hline
\end{tabular}

On the basis of these observations, it is suggested that the $\mathrm{Zn}(\mathrm{II})$ complexes show an octahedral geometry (Fig 2). The two Schiff base moieties acting as tridentate ligands, accommodate themselves around the $\mathrm{Zn}(11)$ metal atom in such a way that a stable chelate ring is formed thus stabiliising the $\mathrm{Zn}(\mathrm{II})$ chelate. 


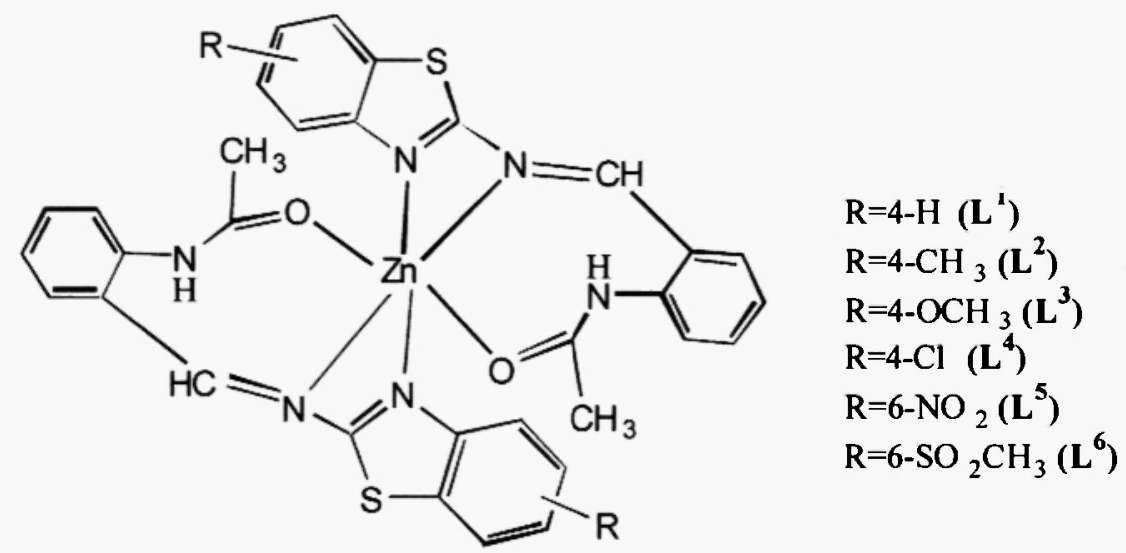

(Fig. 2) Proposed structure of the $\mathrm{Zn}(\mathrm{II})$ complex.

\section{Antibacterial properties}

The title Schiff bases and their $\mathrm{Zn}$ (II) chelates were evaluated for their antibacterial activity against the strains Escherichia coli (a), Staphylococcus aureus (b) and. Pseudomonas aeruginosa (c). The compounds were tested at a concentration of $30 \mu \mathrm{g} / 0.01 \mathrm{~mL}$ in DMF solution using the paper disc diffusion method. The susceptibility zones were measured in diameter $(\mathrm{mm})$ and the results are reproduced in Table 5 . The susceptibility zones measured were the clear zones around the discs killing the bacteria.

All the Schiff bases and their $\mathrm{Zn}$ (II) complexes individually exhibited varying degrees of inhibitory effects on the growth of the tested bacterial species. The antibacterial results evidently show that the activity of the Schiff base compounds became more pronounced when coordinated to the $\mathrm{Zn}$ (II) metal. Our previous studies have suggested ${ }^{33}$ that in the chelated complex, the positive charge of the metal ion is partially shared with the donor atoms and there is $\pi$-electron delocalization over the whole chelate ring. This increases the lipophilic character of the metal chelate and favors its permeation through lipoid layers of the bacterial membranes. Apart from this, other factors such as solubility, conductivity and dipole moment, influenced by the presence of the metal ion, may also be the possible reasons for increasing this activity of the ligands upon chelation.

Table 4. Antibacterial Activity Data.

\begin{tabular}{|c|c|c|c|}
\hline $\begin{array}{c}\text { Schiff base/ } \\
\text { Complex }\end{array}$ & $\begin{array}{c}\text { M i c o b } \\
\text { (a) }\end{array}$ & i a l $\mathbf{~ S ~}$ & p e c i e s \\
\hline $\mathbf{L}^{3}$ & ++ & +++ & ++ \\
\hline $\mathbf{L}^{2}$ & ++ & ++ & + \\
\hline $\mathbf{L}^{3}$ & + & ++ & ++ \\
\hline $\mathrm{L}^{4}$ & ++ & ++ & +++ \\
\hline $\mathrm{L}^{3}$ & +++ & + & ++ \\
\hline $\mathrm{L}^{6}$ & ++ & ++ & ++ \\
\hline$(1)$ & +++ & ++++ & +++ \\
\hline$(2)$ & +++ & +++ & ++ \\
\hline$(3)$ & ++++ & ++ & +++ \\
\hline$(4)$ & +++ & ++++ & +++ \\
\hline$(5)$ & +++ & +++ & +++ \\
\hline$(6)$ & ++++ & +++ & ++++ \\
\hline
\end{tabular}

$(\mathrm{a})=$ Escherichia coli, $(\mathrm{b})=$ Staphylococcus aureus, $(\mathrm{c})=$ Pseudomonas aeruginos Inhibition zone diameter mm (\% inhibition):,$+ 6-10(27-45 \%) ;++, 10-14$

$(45-64 \%) ;+++, 14-18(64-82 \%) ;++++, 18-22(82-100 \%)$. Percent inhibition values are relative to inhibition zone $(22 \mathrm{~mm})$ of the most active compound with $100 \%$ inhibition.

\section{REFERENCES}

1. B. Dash, M. Patra and S. Praharaj, Ind. J. Chem, 19B, 894 (1980).

2. M. Patra, S. K. Mahapatra and B. Dash, J. Ind. Chem. Soc, 51, 1031 (1974).

3. A. Scozzafava, F. Briganti, G. Mincione, L. Menabuoni, F. Mincione, C.T. Supuran, J. Med. Chem., 42, 3690-3700 (1999)

4. A. Scozzafava, F. Briganti, M.A. Ilies, C.T. Supuran, J. Med. Chem., 43, 292-300 (2000).

5. R. L. Duta and M. M. Hossain, J. Sci. Ind. Res, 44, 635 (1985).

6. C. L. Clein, E. D. Stevens, C. J. O'Connor, R. J. Mejestes and L. M. Trefonas, Inorg. Chim. Acta, 70, 151 (1983). 
7. P. B. Maravalli, S. D. Dhumwad and T. R. Goudar, Synth. React. Inorg. Met-Org. Chem, 29, 525 (1999).

8. A. A. A. Emara, Synth. React. Inorg. Met-Org. Chem, 29, 87 (1999).

9. a) Z. H. Chohan and S. K. A. Sherazi, Synth. React. Inorg. Met-Org. Chem, 29, 105 (1999). b) Z. H. Chohan and C. T. Supuran, Main Group Metal Chem, 24, 7 (2001). c) Z. H. Chohan and S. Mushtaq, Pak. J. Pharmaceut. Sci, 13, 21 (2000). d) Z. H. Chohan, A. Munawar and C. T. Supuran, Metal Based Drugs, 8, 137 (2001). e) Z. H. Chohan and M. Praveen, Appl. Organomet. Chem, 15, 617 (2001). f) Z. H. Chohan, Synth. React. Inorg. Met-Org. Chem, 31, 1 (2001). g) Z. H. Chohan, M. Jaffery and C. T. Supuran, Metal Based Drugs, 8, 95 (2001).

10. M. Tastekin, A. Kenar, O. Atakol, M. N. Tahir and D. Ulku, Synth. React. Inorg. Met-Org. Chem, 28, $1727(1998)$.

11. X. Zhang, Y. Zhang, L. Yang, R. Yang and D. Jin, Synth. React. Inorg. Met-Org. Chem, 30, 45 (2000).

12. M. Lashanizadegan and D. M. Boghaei, Synth. React. Inorg. Met-Org. Chem, 30, 89 (2000).

13. M. Gomez-Bosquet, V. Moreno, M. Font-Bardia and X. Solans, Metal Based Drugs, 5, 161 (1998).

14. D. R. Williams, "The Metals of Life", Van Nostrand, London (1971).

15. H. Sigel and D. B. McCormick, Acct. Chem. Res, 3, 201 (1970).

16. M. Dixon and E. C. Webb, "Enzymes", Green and Co, London (1964).

17. B. L. Vallee and J. E. Coleman, Compr. Biochem, 12, 165 (1964).

18. D. R. Williams, Chem. Rev, 72, 203 (1972).

19. A. Albert, Aust. J. Sci, 30, 1 (1967).

20. S. Kirschner, Y. K. Wei, D. Francis and J. G. Bergman, J. Med. Chem, 9, 369 (1966).

21. J. A. Crim and H. G. Petering, Cancer. Res, 27, 1109 and 1278 (1967).

22. S. E. Luria, Cancer. Res, 20,669 (1960).

23. M. Huang, A. Maynard, J. A. Turpin, L. Graham, G. M. Janini, D. G. Covell and W. G. Rice, J. Med. Chem, 41, 1371 (1998).

24. W. Beerheide, M. M. Sim, Y. J. Tan, H. U. Bernard and A. E. Ting, Bioorg. Med. Chem, 8, 2549 (2000).

25. M. E. Heim, "Metal Complexes in Chemotherapy", Verlag Chemie, Weinheim (1993).

26. a) Z. H. Chohan and S. Kausar, Chem. Pharm. Bull, 41, 951 (1993). b) Z. H. Chohan and S. Kausar, Chem. Pharm. Bull, 40, 2552 (1992). c) Z. H. Chohan and A. Rauf, J. Inorg. Biochem, 46, 41 (1992).

27. W. J. Geary, Coord. Chem. Rev, 7, 81 (1971).

28. L. J. Bellamy, "The Infrared Spectra of Complex Molecules", $3^{\text {rd }}$ Ed, Methuen, London (1966).

29. M. Yongxiang, Z. Zhengzhi, M. Yun and Z. Gang, Inorg. Chim. Acta, 165, 185 (1989).

30. K. Nakamoto, "Infrared Spectra of Inorganic and Coordination Compounds", $2^{\text {nd }}$ Ed, Wiley Interscience, New York (1970).

31. D. J. Pasto and C. R. Johnson, "Organic Structure Determination", $4^{\text {th }}$ Ed, Prentice-Hall, International, Inc, U K (1969).

32. Z. Hong-Yun, C. Dong-Li, C. Pei-Kun, C. De-Ji, C. Guang-Xia and Z. Hong-Quan, Polyhedron, 11, 2313 (1992).

33. A. B. P. Lever, "Inorganic Electronic Spectroscopy", Elsevier, Amsterdam (1984).

34. B. N. Figgis, "Introduction to Ligand Fields", J. Wiley, New York (1976).

35. a) Z. H. Chohan, M. A. Farooq and M. S. Iqbal, Metal Based Drugs, 7, 177 (2000). b) Z. H. Chohan and M. Praveen, Appl. Organomet. Chem, 14, 376 (2000). c) Z. H. Chohan and M. F. Jaffery, Metal Based Drugs, 7, 265 (2000).

Received: February 26, 2002 - Accepted: March 4, 2002 Accepted in publishable format: March 5, 2002 